\title{
Cost of inpatient rehabilitation for children with moderate to severe traumatic brain injury
}

Jia Hui Teo, ${ }^{1}$ MBBS (Melb), MRCPCH (UK), MMed (Paeds) (S'pore), Shu-Ling Chong, ${ }^{2}$ MBBS (S'pore), MRCPCH (UK), MPH (US),

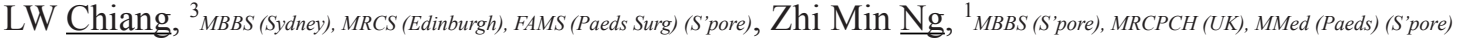

\begin{abstract}
Aim: To evaluate the cost of inpatient rehabilitation for children with moderate to severe traumatic brain injury (TBI). Secondary aim was to identify factors associated with high inpatient rehabilitation cost.

Method: Retrospective review of a tertiary hospital's trauma registry was performed from 2011-2017. All patients aged 16 years or younger who sustained TBI with Glasgow Coma Scale $\leq 13$ were included. Data on patient demographics, mechanism and severity of injury, hospital duration and inpatient rehabilitation cost were collected. We performed a regression analysis to identify factors associated with high rehabilitation cost.

Results: There were a total of 51 patients. The median duration of inpatient rehabilitation was 13.5 days (interquartile range [IQR] 4-35), amounting to a median cost of SGD8,361 (IQR 3,543-25,232). Daily ward costs contributed the most to total inpatient rehabilitation cost. Those with severe TBI had longer duration of inpatient rehabilitation that resulted in higher cost of inpatient rehabilitation. Presence of polytrauma, medical complications, post-traumatic amnesia and TBI post-non-accidental injury (NAI) were associated with higher cost of inpatient rehabilitation.

Conclusion: The cost of inpatient rehabilitation for paediatric patients post-TBI is significant in Singapore. Patients with TBI secondary to NAI had significantly higher cost of inpatient rehabilitation. Ways to reduce duration of hospitalisation post-TBI and early step-down care or outpatient rehabilitation should be explored to reduce cost.
\end{abstract}

Ann Acad Med Singap 2021;50:26-32

Keywords: Duration, paediatrics, rehabilitative medicine

\section{INTRODUCTION}

The cost of rehabilitation for children post-traumatic brain injury (TBI) is significant. The annual total healthcare cost of TBI had been estimated to range from USD5.9 billion-76.5 billion. ${ }^{1-3}$ Studies performed in the adult population reported that the direct cost of acute rehabilitation had been relatively similar over a 10 -year period in the US from a median cost of USD53,119 per patient per year in 1993 to USD46,014 per patient per year in $2003 .{ }^{4,5}$ This cost stability could be due to the shorter duration of acute rehabilitation. Acute rehabilitation had been reported to account for at least one-third of the total hospitalisation cost in the paediatric population. ${ }^{6}$

The indirect cost secondary to TBI was also significant. This could be due to loss of employability, burden on caregivers and loss of total family income. However, they had been difficult to quantify and had not been well reported in the literature. ${ }^{7,8}$

The majority of paediatric TBI was reported to be secondary to motor vehicle injury or unintentional fall from height. ${ }^{6,9,10}$ The presence of intracranial haemorrhage and skull fractures were predictive of longer rehabilitation length of stay and hence charges. ${ }^{11}$ Severe TBI, presence of medical complications, longer post-traumatic amnesia and lower functional scores were reported to be associated with higher healthcare costs. $^{5,12-15}$ Specifically, in the paediatric population, TBI with presence of diffuse axonal injury is known to be more severe to require longer duration of rehabilitation. ${ }^{16}$ Some studies have found that TBI secondary to assault did not account for a higher cost. ${ }^{12-14}$

\footnotetext{
${ }^{1}$ Department of Paediatric Medicine, KK Women's and Children's Hospital, Singapore

2 Department of Emergency Medicine, KK Women's and Children's Hospital, Singapore

${ }^{3}$ Department of Paediatric Surgery, KK Women's and Children's Hospital, Singapore

Correspondence: Dr Jia Hui Teo, Department of Paediatric Medicine, KK Women's and Children's Hospital, 100 Bukit Timah Road, Singapore 229899.

Email: jiahui.teo@mohh.com.sg
} 
In the local Singapore adult population, it was reported that patients with TBI received inpatient rehabilitation for about a month, which correlated with a median cost of SGD7,845.50, before taking into account government subsidy. ${ }^{12}$ This cost was largely contributed by bed, board and nursing charges. This was lower compared to the USD46,014 (SGD63,959) reported in a study from the US. ${ }^{5}$

At KK Women's and Children's Hospital in Singapore, children post-TBI start rehabilitation services once they were transferred out from the children's intensive care unit (ICU). Patients were first assessed by the neuro-rehabilitation team consisting of the doctor, physiotherapist, occupational therapist, and speech and language therapist. Rehabilitation goals were set and the therapy sessions then planned. Typically, for a moderate to severe TBI child, daily therapy sessions of 1 hour for each discipline will be given on weekdays. ${ }^{17}$

There is currently no data in Singapore on the cost of inpatient rehabilitation for children post-TBI. This information would be valuable for resource planning and would help policy makers understand the needs of children with TBI. The primary aim of this study was to evaluate the cost of inpatient rehabilitation for children with moderate to severe TBI. The secondary aim was to identify factors associated with a high inpatient rehabilitation cost.

\section{METHODS}

\section{Patients and data}

We performed a retrospective cohort study using data from the trauma registry in KK Women's and Children's Hospital (KKH). Patients with International Classification of Diseases diagnostic codes that included TBI from 2011-2017 were screened. We included all patients less than or equal to 16 years old with admitting Glasgow Coma Scale (GCS) of 13 or less. We excluded patients with early mortality (died within the first week of hospital stay) or any patients who did not receive any inpatient rehabilitation.

We collected data including demographic profile, admitting GCS, severity of TBI, aetiology of injury and type of injury on the first computed tomography (CT) brain scan. Severity of TBI was based on admitting GCS: Moderate TBI was defined as any patient with admitting GCS of 9-13. Severe TBI was defined as any patient with admitting GCS of 3-8. ${ }^{18}$ Aetiology of injury was categorised into: motor vehicle accident (MVA), fall from height, non-accidental injury (NAI) and others. Type of injury of first CT scan would include diffuse axonal injury (DAI), lobar contusion, intracranial haemorrhage (extradural haemorrhage, subdural haemorrhage and subarachnoid haemorrhage), skull fracture, or mixed (both DAI and intracranial haemorrhage, or lobar contusions with intracranial haemorrhage).

We also collected information regarding duration of hospital stay, ICU stay, presence of polytrauma, posttraumatic amnesia (PTA) and medical complications. PTA was defined as a partial or total loss of the ability to recall events that have occurred during the period immediately preceding brain injury. ${ }^{19}$ PTA was scored with the use of Westmead PTA scale in our institution. However, the score was not available for all patients, hence only information regarding presence or absence of PTA was obtained.

\section{Outcomes}

Data on total hospitalisation cost and inpatient rehabilitation cost were collected. Inpatient rehabilitation costs included the total gross cost of general ward stay, costs of consumables (including diapers, wound dressings, intravenous cannulas and urinary catheters), rehabilitative services (including orthoses and walking aids) and nursing costs. This excluded the cost incurred from children's ICU stay and surgical cost. All financial data were obtained from the department of finance. In order to negate the effects of national healthcare subsidies on different ward classes, non-subsidised costs were used. Duration of inpatient rehabilitation was derived from total length of hospital stay, excluding length of stay in ICU. Outpatient rehabilitation cost was taken as total outpatient cost over a 6-month period from discharge.

The WeeFIM instrument, a measure of functional abilities in patients aged 6 months -18 years, was used to measure functional outcome at discharge in this study. WeeFIM had been reported to be the preferred functional assessment tool for paediatric patients post-acquired brain injury. ${ }^{20}$ WeeFIM incorporated 18 items across domains of mobility (self-care, transfers and locomotion) and cognition (communication and social cognition), each rated on a 7-level ordinal scale. The maximum rating of 7 on this scale represented complete independence and the minimum rating of 1 represented total assistance. The total rating for all 18 items could range from 18 to a maximum of 126 . In our institution, the WeeFIM assessment tool had been used for TBI in children since 2011, performed at the initiation of rehabilitation, at discharge and subsequently on follow-up. However, in our study, we collected primarily data on WeeFIM scores at discharge. 


\section{Statistical analysis}

Patient demographics were analysed as frequencies. Categorical data were presented as number and frequency. Continuous data were presented as median with interquartile range (IQR) or mean with standard deviation (SD), depending on normality of data. Comparison of continuous variables between groups was performed using Mann-Whitney test if there were binary groups, or Kruskal Wallis test if there were more than 2 groups. Linear regression analysis was performed on variables that were decided clinically on the basis that these would contribute to the cost of inpatient rehabilitation and total hospitalisation costs, in SGD1,000 increment. We present the regression analysis using point estimates and $95 \%$ confidence intervals (95\% CI). Statistical significance was taken as $P<0.05$ for all tests. Statistical analysis was performed using SPSS Statistics version 26.0 (IBM Corp, Armonk, US).

\section{RESULTS}

\section{Clinical demographics}

There were 61 patients obtained from the trauma registry with injuries occurring from 1 January 2011 to 31 October 2017. Of this number, only 51 met the inclusion criteria. The mean age of injury was 8.0 (SD 4.5) years, with $65 \%$ male. There were 26 cases $(51 \%)$ with moderate TBI and 25 cases (49\%) with severe TBI. The majority $(27,53 \%)$ suffered TBI from MVA, with intracranial haemorrhage as the initial CT scan finding (31, 61\%). Twenty-eight $(55 \%)$ cases had polytrauma, $12(24 \%)$ had medical complications and 22 (43\%) had PTA (Table 1).

\section{Outcomes}

Patients with TBI had a median hospitalisation duration of 20 days (IQR 7.0-45.0). The median length of stay in ICU was 5 days (IQR 1.0-11.0). The median duration of inpatient rehabilitation was 13.5 days (IQR 4-35), which corresponded to a median cost of SGD8,361 (IQR 3,543-25,232). At discharge, patients with moderate TBI had a higher median WeeFIM score compared to those with severe TBI (125 [IQR 97-126] versus 83 [IQR 30-95], $P=0.021$ ).

\section{Duration of stay and inpatient rehabilitation cost}

When comparing the duration of inpatient rehabilitation between severe TBI and moderate TBI, those with severe TBI had longer duration of inpatient rehabilitation (30.5 days [IQR 10.2-38.7] vs 5.5 days [IQR 4.0-17.2], $P=0.006)$. Severe TBI resulted in higher inpatient rehabilitation costs over 6 months compared to moderate TBI (SGD17,764 [IQR 7,715.5-30,260.5] vs SGD4,912.1 [IQR 1,972.8-13,932.7], $P=0.003$ ).

Severe TBI also resulted in higher outpatient rehabilitation over 6 months when compared with moderate TBI (S $\$ 3,090.7$ [IQR 1,599-14,291.3] vs SGD1,300 [IQR 504-2,396.5], $P=0.021$ ). The total cost of hospitalisation for patients with severe TBI was higher compared to those with moderate TBI [SGD46,825 (IQR 24,386.9-77,600.6) vs SGD21,051.2 (IQR 9,492.8-50,865.9), $P=0.018$ ] (Table 2). The cost of inpatient rehabilitation ranged from $23-38 \%$ of total hospitalisation cost.

\section{Components of inpatient rehabilitation cost}

The majority of the inpatient rehabilitation cost was contributed by daily ward charges with median costs of SGD3,258 (IQR 1,116-9,036) (Table 3). This was approximately $40 \%$ of the inpatient rehabilitation costs. Rehabilitative services comprised $17 \%$ of the total inpatient rehabilitation cost. Other contributing factors were daily treatment costs, consumables, ward

Table 1. Baseline characteristics

\begin{tabular}{lc}
\hline Age, mean (SD), years & $8.0(4.5)$ \\
& $\mathrm{n}(\%)$ \\
\hline Male & $33(65)$ \\
Race & \\
Chinese & $30(59)$ \\
Malay & $10(20)$ \\
Indian & $6(11)$ \\
Others & $5(10)$ \\
Aetiology & \\
Motor vehicle accident & $27(53)$ \\
Fall & $18(35)$ \\
Non-accidental injury & $3(6)$ \\
Others & $3(6)$ \\
Injury type & \\
DAI & $6(12)$ \\
Lobar contusion & $4(8)$ \\
ICH (SDH/EDH/SAH) & $31(61)$ \\
Mixed (DAI/ICH or lobar contusion/ICH) & $9(17)$ \\
Skull fracture & $1(2)$ \\
Polytrauma & \\
Yes & $28(55)$ \\
Medical complications & \\
Yes & \\
Post-traumatic amnesia & \\
Yes & \\
\hline DAl: & \\
\hline
\end{tabular}

DAI: diffuse axonal injury; ICH: intracranial haemorrhage; SDH: subdural haemorrhage; EDH: extradural haemorrhage; SAH: subarachnoid haemorrhage; SD: standard deviation a 1 hit by golf club, 1 fell from bicycle, 1 hit by rocks during earthquake 
Table 2. Primary outcomes

\begin{tabular}{|c|c|c|c|}
\hline Median (IQR) & Moderate TBI $(n=26)$ & Severe TBI $(n=25)$ & $P$ value \\
\hline Total duration of hospitalisation, days & $10.0(5.0-24.2)$ & $38.0(20.0-52.5)$ & 0.002 \\
\hline Duration of inpatient rehabilitation, days & $5.5(4.0-17.2)$ & $30.5(10.2-38.7)$ & 0.006 \\
\hline Cost of inpatient rehabilitation, SGD & $4,912.1(1,972.8-13,932.7)$ & $17,764.0(7,715.5-30,260.5)$ & 0.003 \\
\hline Cost of total hospitalisation, SGD & $21,051.2(9,492.8-50,865.9)$ & $46,825.0(24,386.9-77,600.6)$ & 0.018 \\
\hline Cost of outpatient rehabilitation, ${ }^{a}$ SGD & $1,300.0(504.0-2,396.5)$ & $3,090.7(1,599.1-4,291.3)$ & 0.021 \\
\hline
\end{tabular}

IQR: interquartile range; TBI: traumatic brain injury

${ }^{a}$ Cost of outpatient rehabilitation measured at 6 months from time of discharge

Table 3. Components of inpatient hospitalisation cost

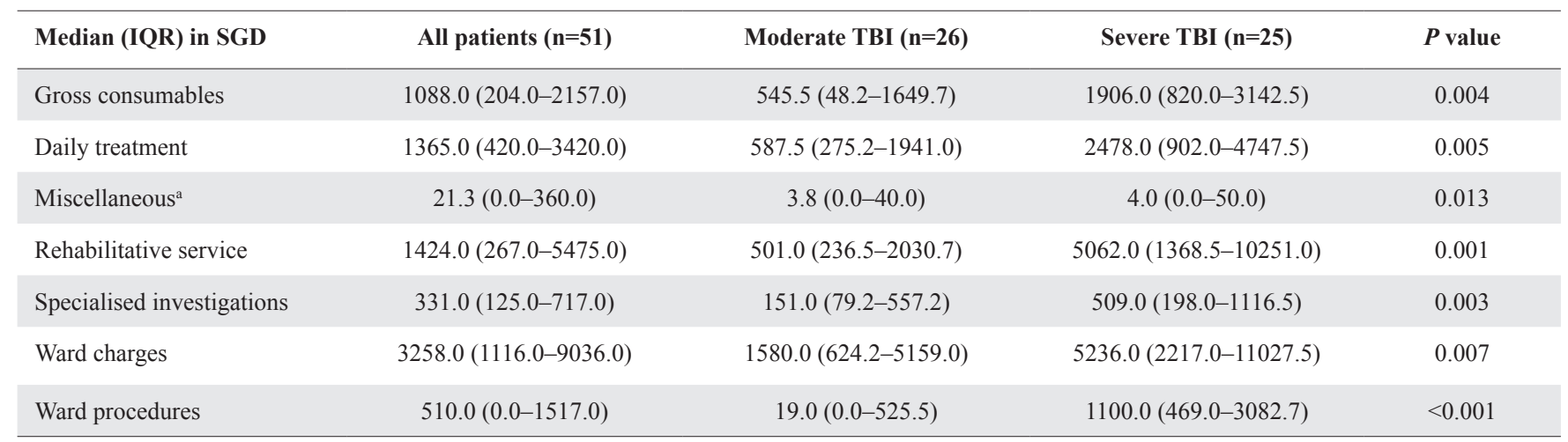

IQR: interquartile range; TBI: traumatic brain injury

${ }^{a}$ Median (Range)

procedures, specialised investigations and miscellaneous non-treatment related costs, in decreasing order. The difference in cost for each component of inpatient rehabilitation between patients with severe and moderate TBI was statistically significant $(P<0.05)$.

\section{Factors associated with higher inpatient rehabilitation cost}

In terms of aetiology, patients with TBI post-NAI had significantly higher median costs of inpatient rehabilitation (SGD130,444.6 [IQR 42,894-222,420]), as compared to TBI secondary to motor vehicle accident, falls and other causes of injury (SGD13,333 [IQR 4,459.2-21,949], SGD3,821 [IQR 1,857.611,222.3], and SGD12,434.3 [IQR 1,761-7,971], respectively, $P=0.008$ ).

Patients with polytrauma also had higher median costs of inpatient rehabilitation compared to those without polytrauma (SGD15,563.5 [IQR 5,805.7-27,481.7] vs SGD4,507 [IQR 2,043.4-9,201] $\mathrm{p}=0.009$ ). Those with presence of PTA also had higher costs of inpatient rehabilitation (SGD19,907.5 [IQR 8,975.0-37,151.2] vs SGD3,958.5 [IQR 1,831.6-7,961.2], $\mathrm{p}<0.001)$.

Of the 12 patients with medical complications, 5 had pneumonia, 3 had seizures, 2 had sepsis, 1 with urethral ulcer and 1 with pituitary dysfunction. Patients with medical complications did not have a statistically longer hospitalisation duration or longer inpatient rehabilitation duration, compared to those without complications. However, those with medical complications had a higher median cost of inpatient rehabilitation than those without (SGD17,866 [IQR 8,361-32,950] vs SGD5,475 [IQR 2,809.9-15,563.5], $P=0.016$ ).

The difference in type of injury on initial CT scan did not affect the duration of inpatient rehabilitation $(P=0.08)$ or cost of inpatient rehabilitation $(P=0.123)$.

When factors such as age, gender, severity of TBI, mechanism of injury, presence of polytrauma and PTA were considered on multivariate analysis, only TBI secondary to NAI resulted in significantly higher cost of inpatient rehabilitation and total hospitalisation (Table 4). 
Table 4. Multivariate analysis of factors affecting cost of inpatient rehabilitation and total hospitalisation ${ }^{\mathrm{a}}$

\begin{tabular}{|c|c|c|c|c|c|c|}
\hline & \multicolumn{3}{|c|}{ Inpatient rehabilitation cost } & \multicolumn{3}{|c|}{ Total hospitalisation cost } \\
\hline & Coefficient & $95 \% \mathrm{CI}$ & $P$ value & Coefficient & $95 \% \mathrm{CI}$ & $P$ value \\
\hline Age at injury & 0.72 & $-1.11-2.54$ & 0.431 & 3.97 & $-0.61-8.55$ & 0.087 \\
\hline Female & -2.83 & $-15.54-9.88$ & 0.655 & -18.41 & $-50.34-13.52$ & 0.250 \\
\hline TBI secondary to motor vehicle accident & 3.64 & $-9.44-16.73$ & 0.576 & 21.03 & $-11.84-53.91$ & 0.203 \\
\hline TBI secondary to NAI & 164.93 & $132.83-197.03$ & $<0.001$ & 211.50 & $130.86-292.15$ & $<0.001$ \\
\hline Presence of medical complications & 3.88 & $-11.76-19.52$ & 0.618 & 16.78 & $-22.52-56.08$ & 0.393 \\
\hline Presence of polytrauma & -0.50 & $-13.56-12.56$ & 0.939 & 15.59 & $-17.22-48.40$ & 0.342 \\
\hline Presence of post-traumatic amnesia & 8.01 & $-6.41-22.42$ & 0.268 & -2.71 & $-38.94-33.52$ & 0.880 \\
\hline Severe TBI & 2.25 & $-10.90-15.41$ & 0.731 & -6.71 & $-39.77-26.35$ & 0.683 \\
\hline
\end{tabular}

TBI: traumatic brain injury; NAI: non-accidental injury; CI: confidence interval

${ }^{a}$ Estimates are given per SGD1,000

\section{DISCUSSION}

We found that rehabilitation cost is significant for children with TBI and that severity of TBI, presence of polytrauma, medical complications, PTA and TBI secondary to NAI were associated with high costs.

Our study found more boys than girls had TBI, most of them of school-going age. Presence of intracranial haemorrhage and polytrauma were commonly seen in TBI secondary to motor vehicle accidents in children, a situation similar to the adult population. ${ }^{13}$

In our study, severity of TBI was associated with higher cost. This was likely contributed by inpatient rehabilitation (median duration of 1 month), as compared to less than a week for patients with moderate TBI. This difference was significant, and more so when translated to actual cost of inpatient rehabilitation. Those with severe TBI had to pay at least 3 times more than those with moderate TBI for inpatient rehabilitation. This association between severity of TBI and cost of inpatient rehabilitation had been previously reported in adults. ${ }^{12}$ In our study population, the cost of inpatient rehabilitation accounted for close to one-third of the total hospitalisation for patients with moderate to severe TBI. The majority of the total hospitalisation cost was secondary to surgery or ICU stay. This was similar to a previous study that reported that rehabilitation charges (mean charges USD560) were about one-third of the daily hospitalisation cost (USD1,562.2). ${ }^{5}$

However, the largest contributing factor to inpatient rehabilitation costs was daily ward charges, a finding similar to the report on the adult local population. ${ }^{12}$ It is important to note that the cost of rehabilitative services was not the highest contributing factor to inpatient rehabilitation cost.

We reported that presence of polytrauma, medical complications and PTA were associated with higher cost of inpatient rehabilitation. Presence of polytrauma, medical complications and PTA were also previously reported to be predictors of higher cost of care (total hospitalisation and rehabilitation costs) post-TBI. This was attributed to the likelihood of a need for intensive care and indicative of severity of TBI if patients had polytrauma, medical complications or PTA. It was also reported that the duration of PTA was the strongest predictor of various types of costs following TBI. ${ }^{12,14}$ However, as our study did not collect information regarding the duration of PTA, we were unable to make this similar conclusion. The presence of PTA alone may not be useful as a factor associated with higher inpatient rehabilitation cost, since presence of PTA itself could be associated with severity of TBI.

Our study found that patients with severe TBI had lower functional scores at discharge. It could be extrapolated that their functional scores at the initiation of rehabilitation were likely lower. As such, they may have required longer duration of inpatient rehabilitation, resulting in higher costs of rehabilitation for this group of patients. However, information of the functional scores at initiation of rehabilitation was not collected in this study.

In multivariate analysis, the only factor that remained significant after accounting for mechanism of injury, age, gender, severity of TBI, presence of polytrauma, medical complications and PTA was NAI. The difference in the 
cost and duration of inpatient rehabilitation was statistically significant. In addition, when we analysed our data without patients with TBI secondary to NAI, results were similar.

In our study with 3 patients with TBI post-NAI, the case that had the longest length of stay of 354 days had moderate TBI. This patient had no polytrauma and did not require intensive care. The main reasons for prolonged hospitalisation were due to multiple nosocomial infections, mainly upper respiratory tract infections or gastroenteritis, and the involvement of social services to determine safe placement in view of NAI. As such, this patient incurred a total cost of inpatient hospitalisation amounting to SGD222,420.

We have previously reported that patients with TBI secondary to NAI had poorer functional outcomes at initiation of rehabilitation. ${ }^{21}$ This could have resulted in a longer duration of stay for inpatient rehabilitation. Legal issues and placement concerns would also contribute to a longer hospitalisation and higher cost related to the inpatient stay. Given the high cost of management of TBI with long-term sequelae, it would be important to look at education and preventive measures to reduce the incidence of TBI in children, particularly NAI. ${ }^{22}$

\section{Strengths and limitations}

There were several limitations with this study. Given that this was a retrospective study, information bias was present and not all data collected were complete (such as WeeFIM scores and duration of PTA). We did not have data on specific breakdown of cost for each component of inpatient rehabilitation-be it cost of allied health consultations or equipment. In terms of type of injury, our study reported injury type based on initial CT scan. Further information on whether brain magnetic resonance imaging was performed for these patients would have been helpful to further classify the injury type.

We did not include patients with mild TBI, which although milder in severity of injury, occurs in a large number of children and may contribute significantly to consumption of resources at the tertiary level. While direct healthcare cost was significant, our study did not look at the indirect healthcare cost of patients with TBI. This would include the loss of employability, increased family expenditure and loss of family income when one parent needed to stop working to help care for the patient.

Despite the limitations, this is the first study to the best of our knowledge to evaluate the cost of inpatient rehabilitation for children with moderate to severe TBI in Singapore. We have highlighted the vulnerable groups that required intensive resource utilisation. This would be important for future health services research as we seek to improve the care of children with moderate to severe TBI.

\section{CONCLUSION}

The direct cost associated with post-paediatric TBI rehabilitation is significant, especially for patients with TBI with polytrauma, medical complications, PTA and TBI secondary to NAI. A majority of the inpatient cost was contributed by daily ward charges. Therefore, ways to reduce hospitalisation duration post-TBI and early step-down care or outpatient rehabilitation should be explored to reduce cost. Further studies are also needed to look at indirect costs to evaluate the actual socio-economic burden of paediatric TBI.

\section{REFERENCES}

1. Access Economics. The economic cost of spinal cord injury and traumatic brain injury in Australia. Canberra: Access Economics; 2009.

2. Olesen J, Gustavsson A, Svensson M, et al. The economic cost of brain disorders in Europe. Eur J Neurol 2012;19:155-62.

3. Finkelstein EA, Corso PS, Miller TR. The incidence and economic burden of injuries in the United States. New York: Oxford University Press; 2006.

4. Kreutzer JS, Kolakowsky-Hayner SA, Ripley D, et al. Charges and lengths of stay for acute and inpatient rehabilitation treatment of traumatic brain injury 1990-1996. Brain Inj 2001;15:763-74.

5. Mayer NH, Pelensky J, Whyte $J$ et al. Characterization and correlates of medical and rehabilitation charges for traumatic brain injury during acute rehabilitation hospitalization. Arch Phys Med Rehabil 2003;84:242-8.

6. Jaffe KM, Massagli TL, Martin KM et al. Pediatric traumatic brain injury: acute and rehabilitation costs.Arch Phys Med Rehabil 1993;74:681-6.

7. Khan F, Baguley IJ, Cameron ID. Rehabilitation after traumatic brain injury. Med J Aust 2003;178:290-5.

8. Wade SL, Taylor HG, Drotar D et al. Family burden and adaptation during the initial year after traumatic brain injury in children. Pediatrics 1998;102:110-6.

9. Tabish A, Lone NA, Afzal WM, et al. The incidence and severity of injury in children hospitalised for traumatic brain injury in Kashmir. Injury 2006;37:410-5.

10. Shi J, Xiang H, Wheeler K, et al. Costs, mortality likelihood and outcomes of hospitalized US children with traumatic brain injuries. Brain Inj 2009;23:602-11.

11. Cowen TD, Meythaler JM, DeVivo MJ, et al. Influence of early variables in traumatic brain injury on functional independence measure scores and rehabilitation length of stay and charges. Arch Phys Med Rehabil 1995;76:797-803.

12. Chua KS, Earnest A, Chiong Y, et al. Characteristics and correlates of rehabilitation charges during inpatient traumatic brain injury rehabilitation in Singapore. J Rehabil Med 2010;42:27-34.

13. Ponsford JL, Spitz G, Cromarty F, et al. Costs of care after traumatic brain injury. J Neurotrauma 2013;30:1498-505. 
14. Spitz G, McKenzie D, Attwood D, et al. Cost prediction following traumatic brain injury: model development and validation. J Neurol Neurosurg Psychiatry 2016;87:173-80.

15. Jaffe KM. Pediatric trauma rehabilitation: a value-added safety net. J Trauma 2008;64:819-23.

16. Arango-Lasprilla JC, Ketchum JM, Cifu D, et al. Predictors of extended rehabilitation length of stay after traumatic brain injury. Arch Phys Med Rehabil 2010;91:1495-504.

17. Yen HL, Wong JT. Rehabilitation for traumatic brain injury in children and adolescents. Ann Acad Med Singap 2007;36:62-6.

18. Teasdale G, Jennett B. Assessment of coma and impaired consciousness. A practical scale. Lancet 1974;2:81-84.
19. Cartlidge NEF. Head Injury (Major Problems in Neurology). London, England: WB Saunders; 1981.

20. Williams KS, Young DK, Burke GAA, et al. Comparing the WeeFIM and PEDI in neurorehabilitation for children with acquired brain injury: A systematic review. Dev Neurorehabil 2017;20:443-51.

21. Hwang SY, Ong JW, Ng ZM, et al. Long-term outcomes in children with moderate to severe traumatic brain injury: a single-centre retrospective study. Brain Inj 2019;33:1420-4.

22. Ong I. Prevention: The cure for child head injuries, 1 March 2017. Available at: http://www.kkh.com.sg/news/patient-care/preventionthe-cure-for-head-injuries. Accessed on 20 August 2020. 